DRAFT VERSION -, 2017

Preprint typeset using $\mathrm{L}^{\mathrm{A}} \mathrm{T}_{\mathrm{E}} \mathrm{X}$ style emulateapj v. 01/23/15

\title{
BROADBAND X-RAY SPECTRAL ANALYSIS OF THE DOUBLE-NUCLEUS LUMINOUS INFRARED
} GALAXY MRK 463

\author{
Satoshi Yamada ${ }^{1}$, Yoshiniro Ueda ${ }^{1}$, Saeko Oda $^{1}$, Atsushi Tanimoto ${ }^{1}$, Masatoshi Imanishi $^{2,3}$, \\ Yuichi Terashima ${ }^{4}$, AND Claudio RicCI ${ }^{5,6,7}$ \\ ${ }^{1}$ Department of Astronomy, Kyoto University, Kitashirakawa-Oiwake-cho, Sakyo-ku, Kyoto 606-8502, Japan; \\ styamada@kusastro.kyoto-u.ac.jp \\ ${ }^{2}$ National Astronomical Observatory of Japan, 2-21-1 Osawa, Mitaka, Tokyo 181-8588, Japan \\ ${ }^{3}$ Department of Astronomy, School of Science, SOKENDAI (The Graduate University for Advanced Studies), 2-21-1 Osawa, Mitaka, \\ Tokyo 181-8588, Japan \\ ${ }^{4}$ Department of Physics, Ehime University, Matsuyama 790-8577, Japan \\ ${ }^{5}$ Núcleo de Astronomía de la Facultad de Ingeniería, Universidad Diego Portales, Av. Ejército Libertador 441, Santiago, Chile \\ ${ }^{6}$ Kavli Institute for Astronomy and Astrophysics, Peking University, Beijing 100871, China \\ ${ }^{7}$ Chinese Academy of Sciences South America Center for Astronomy, Camino El Observatorio 1515, Las Condes, Santiago, Chile \\ Draft version --, 2017
}

\section{ABSTRACT}

We present a broadband $(0.4-70 \mathrm{keV})$ X-ray spectral analysis of the luminous infrared galaxy (LIRG) system Mrk 463 observed with Nuclear Spectroscopic Telescope Array (NuSTAR), Chandra, and XMMNewton, which contains double active galactic nuclei (AGNs) (Mrk 463E and Mrk 463W) with a separation of $\sim 3.8 \mathrm{kpc}$. Detecting their transmitted hard X-ray $>10 \mathrm{keV}$ continua with $N u S T A R$, we confirm that Mrk 463E and Mrk 463W have AGNs with intrinsic luminosities of $(1.6-2.2) \times 10^{43}$ and $(0.5-0.6) \times 10^{43} \mathrm{erg} \mathrm{s}^{-1}(2-10 \mathrm{keV})$ obscured by hydrogen column densities of $8 \times 10^{23}$ and 3 $\times 10^{23} \mathrm{~cm}^{-2}$, respectively. Both nuclei show strong reflection components from cold matter. The luminosity ratio between X-ray $(2-10 \mathrm{keV})$ and [O IV] $25.89 \mu \mathrm{m}$ of Mrk $463 \mathrm{E}$ is $\sim 5$ times smaller than those of normal Seyfert galaxies, suggesting that the intrinsic SED is X-ray weak relative to the UV luminosity. In fact, the bolometric AGN luminosity of Mrk 463E estimated from $L^{\prime}$-band (3.8 $\mu \mathrm{m}),[\mathrm{O} I V] 25.89 \mu \mathrm{m}$, and [Ne V] $14.32 \mu \mathrm{m}$ lines indicate a large bolometric-to-X-ray luminosity ratio, $\kappa_{2-10 \mathrm{keV}} \approx 110-410$, and a high Eddington ratio, $\lambda_{\mathrm{Edd}} \sim 0.4-0.8$. We suggest that the merger triggered a rapid growth of the black hole in Mrk 463E, which is not yet deeply "buried" by circumnuclear dust. By contrast, the $L^{\prime}$-band luminosity of Mrk $463 \mathrm{~W}$ is unusually small relative to the X-ray luminosity, suggesting that the Eddington ratio is low $\left(<10^{-3}\right)$ and it might be still in an early phase of mergerdriven AGN activity.

Keywords: black hole physics - galaxies: active - galaxies: individual(Mrk 463) - X-rays: galaxies infrared: galaxies

\section{INTRODUCTION}

It is a widespread belief that supermassive black holes (SMBHs) and galactic bulges have co-evolved by regulating each other's growth (see e.g., Kormendy \& Ho 2013. for a recent review). A key mechanism driving the coevolution is mergers of gas-rich galaxies, which trigger intense star formation (SF) and rapid mass accretion onto the SMBHs,which, during this phase, are observed as active galactic nuclei (AGNs) (e.g., Mihos \& Hernquist 1996, Struck 1999; Hopkins et al. 2008). Dust-rich merging galaxies emit the bulk of their bolometric luminosities in the infrared, and are observed as luminous $\left(L_{\mathrm{IR}}(8-\right.$ $\left.1000 \mu \mathrm{m})=10^{11}-10^{12} L_{\odot}\right)$ or ultra-luminous $\left(L_{\mathrm{IR}} \geq\right.$ $10^{12} L_{\odot}$ ) infrared galaxies (LIRGs and ULIRGs; Sanders \& Mirabel 1996). As the central regions of these objects are deeply obscured by gas and dust, multiwavelength observations are required to understand the nature of the system, including the main energy source (SF or AGN) and the structure of the nucleus. In particular, thanks to their strong penetrating power against obscuration, hard X-ray (>10 keV) observations are very useful to detect hidden AGNs, even Compton-thick ones with column densities of $\log N_{\mathrm{H}} / \mathrm{cm}^{-2}=24-25$. Following earlier studies at energies below $10 \mathrm{keV}$ with $X M M$ -
Newton (e.g., Imanishi et al. 2003; Pereira-Santaella et al. 2011) and Chandra (e.g., Teng et al. 2005; Iwasawa et al. 2011a), the Nuclear Spectroscopic Telescope Array (NuS$T A R)$, the first hard X-ray imaging mission covering the 3-79 keV range (Harrison et al. 2013), is now starting to unveil the nature of heavily obscured AGNs in local U/LIRGs by directly detecting their transmitted component (e.g., Teng et al. 2015, Ricci et al. 2016, Oda et al. 2017, Nardini 2017).

To understand the role of mergers in feeding SMBHs, it is important to investigate the AGN properties as a function of the separation between the two galaxies. In this context, studies of dual AGN systems (e.g., Komossa et al. 2003, Koss et al. 2011; Fabbiano et al. 2011) are of particular interest, since they are considered to be in an earlier stage of mergers than typical U/LIRGs showing highly compact cores. From the 22 and 58 month Swift/BAT hard X-ray survey catalog, Koss et al. (2012) identified 17 dual AGNs. They found that the AGN luminosities increases with decreasing separation. Using $N u S T A R$ observations of local merging galaxies, Ricci et al. (2017) showed that the degree of AGN obscuration increases with the evolutionary stage of mergers; the majority of AGNs in the late-merger galaxies are subject to heavy obscuration for which galactic-scale gas and dust 
could be responsible. Detailed studies of AGN structure in individual dual AGNs based on multiwavelength data could help us to better understand the physical processes induced by mergers. However, currently the number of sources for which this has been possible is very limited.

Mrk $463(z=0.0504)$ is a merging system consisting of Mrk 463E (east) and Mrk 463W (west), which show tidal tails as a result of the galaxy-galaxy interaction (Mazzarella et al. 1991). This is a dual AGN system with the third closest physical separation $(3.8 \mathrm{kpc}$, corresponding to an angular separation of 3.8") following NGC 6240 (1.5 kpc) and Mrk 739 (3.4 kpc) among the Swift/BAT dual-AGN sample compiled by Koss et al. (2012), and hence is an ideal target to link earlier and later stage mergers. Up to present, detailed X-ray spectral analyses have been done only below $10 \mathrm{keV}$. Imanishi \& Terashima (2004) observed it with XMM-Newton and found that the X-ray spectrum of Mrk 463 is heavily obscured with $N_{\mathrm{H}} \sim 3 \times 10^{23} \mathrm{~cm}^{-2}$ showing an iron-K $\alpha$ emission line at $6.4 \mathrm{keV}$. However, the two nuclei were not separated due to the limited angular resolution. Using Chandra, Bianchi et al. (2008) detected AGNs from both Mrk 463E and $463 \mathrm{~W}$, confirming that both spectra are heavily obscured. The Mrk 463 system is classified as a LIRG on the basis of the total infrared luminosity, $L_{\mathrm{IR}}(8-1000 \mu \mathrm{m})=10^{11.8} L_{\odot}$ (Imanishi \& Saito 2014). In the mid-IR band, Mrk $463 \mathrm{E}$ is notably brighter than Mrk 463W (Armus et al. 2004). The Spitzer/IRS spectrum of Mrk 463E was reported in Armus et al. (2004), where high excitation lines such as $[\mathrm{O}$ IV $] 25.89 \mu \mathrm{m}$ and [Ne V] $14.32 \mu \mathrm{m}$ were detected.

In this paper, we perform a broadband X-ray (0.4$70 \mathrm{keV}$ ) spectral analysis of Mrk 463, by simultaneously utilizing the data from NuSTAR, XMM-Newton, and Chandra. Detecting their transmitted hard X-ray $(>10$ $\mathrm{keV}$ ) continua with $N u S T A R$, which are little affected by obscuration, and separating the soft X-ray $(<8 \mathrm{keV})$ spectra of Mrk 463E and Mrk 463W with Chandra, we determine the intrinsic luminosities and absorption column densities of both galaxies with the best accuracy so far, by taking into account the reflection components from circumnuclear matter. This paper is structured as follows. Section 2 describes the X-ray observations and data reduction. In Section 3. we present our spectral analysis using two models: an analytical model and a Monte-Carlo based torus model by Ikeda et al. (2009) In Section 4 , we discuss our results mainly by comparing our results with the mid-IR properties. Section 5 summarizes the conclusions. We adopt the cosmological parameters of $\left(H_{0}, \Omega_{\mathrm{m}}, \Omega_{\Lambda}\right)=\left(70 \mathrm{~km} \mathrm{~s}^{-1} \mathrm{Mpc}^{-1}\right.$, $0.3,0.7)$ and the solar abundances of Anders \& Grevesse (1989) throughout this paper. All errors of the spectral parameters are quoted at $90 \%$ confidence limits for a single parameter of interest.

\section{OBSERVATIONS AND DATA REDUCTION}

The observation log of the X-ray data used in this paper (NuSTAR, Chandra, XMM-Newton) is given in Table1. Details of the data reduction are described below.

\subsection{NUSTAR}

NuSTAR observed Mrk 463 on 2014 January 1 for a net exposure of 23.9 ks. NuSTAR carries two co-aligned grazing incidence telescopes coupled with two focal plane modules A and B (FPMA and FPMB), which are sensitive to X-ray energies of 3-79 keV. The angular resolution of the telescopes is 58" in a half-power diameter (HPD). The data were reduced by using the standard pipeline script (NUPIPELINE) available in the $\mathrm{NuS}$ $T A R$ Data Analysis Software (NuSTARDAS, v1.6.0; part of the HEASOFT distribution as of v6.19) with $N u S$ TAR Calibration Database (CALDB) v20161021. Since Mrk 463E and Mrk 463W cannot be separated by the point spread function of NuSTAR, we produced the total spectrum of the Mrk 463 system by accumulating photon events in a circular region with a radius of 50" centered around the two galaxies. The background was taken from a source-free circular region with a radius of 60 ". The spectra and light curves were extracted with the NUPRODUCTS task. Since the signal-to-noise ratio of the FPMA and FPMB spectra are limited, these spectra were then combined by using ADDASCASPEC to improve the statistics. The spectra were binned to contain a minimum of 50 counts per bin. We tested against the null hypothesis that the $3-24 \mathrm{keV}$ flux is constant via a $\chi^{2}$ test. No significant time variability on a timescale of hours was found at $>90 \%$ confidence limits.

\subsection{Chandra}

Chandra (Weisskopf et al. 2002) observed Mrk 463 on 2004 June 11 with the Advanced CCD Imaging Spectrometer (ACIS: Garmire et al. 2003) for a net exposure of 50.0 ks. Thanks to its excellent angular resolution $(<1$ "), Chandra was able to spatially resolve two galaxies as well as extended X-ray emission surrounding them (Bianchi et al. 2008). We reduced the data using Chandra Interactive Analysis of Observations (CIAO) v4.8.1 and the Calibration Database (CALDB) v4.7.2. The event files were reprocessed with the CIAO CHANDRA_REPRO script. Following Bianchi et al. (2008), we used three extraction regions: two circular regions with a radius of 2" centered on the nuclei of Mrk 463E and Mrk 463W, and one circular region with a radius of 7 " encompassing all soft X-ray extended emission from which the two nucleus regions are excluded. The image in the $0.4-8 \mathrm{keV}$ band is shown in Figure 1. The spectra, the redistribution matrix function (RMF) and the auxiliary response file (ARF) were produced with the task SPECEXTRACT. The spectral bins were grouped to contain no less than 25, 20, and 20 counts for Mrk 463E, Mrk 463W, and the soft extended emission, respectively, so that $\chi^{2}$ statistics is applicable while line features were not smeared out beyond the energy resolution.

\subsection{XMM-Newton}

An XMM-Newton (Jansen et al. 2001) observation of Mrk 463 was performed on 2001 December 22 for a net exposure of $26.8 \mathrm{ks}$. The main instruments of $X M M$ Newton are three European Photon Imaging Cameras (EPICs): PN (Strüder et al. 2001), MOS1, and MOS2 (Turner et al. 2001). The data were reduced in a standard manner with the XMM-Newton Science Analysis System (SAS) v15.0.0. We removed events recorded during background flares, and only utilized those with patterns 0-4 for PN and patterns 0-12 for MOS. The spectra of Mrk 463E, Mrk 463W, and the soft extended emission cannot be separated with XMM-Newton, whose angular resolution is 15 " in HPD. The total source spectra 
Table 1

Observation Log of Mrk 463 Utilized in this Work

\begin{tabular}{|c|c|c|c|c|}
\hline Satellite & ObsID & Start Date(UT) & End Date(UT) & Net exp.(ks) \\
\hline NuSTAR & 60061249002 & 2014-01-01 22:31:07 & 2014-01-02 10:41:07 & 23.9 \\
\hline Chandra (ASIS-S) & 4913 & 2004-06-11 23:06:47 & 2004-06-12 $13: 42: 31$ & 48.8 \\
\hline$X M M$-Newton & 0094401201 & 2001-12-22 03:13:30 & $2001-12-22 \quad 10: 40: 18$ & 25.8 \\
\hline
\end{tabular}

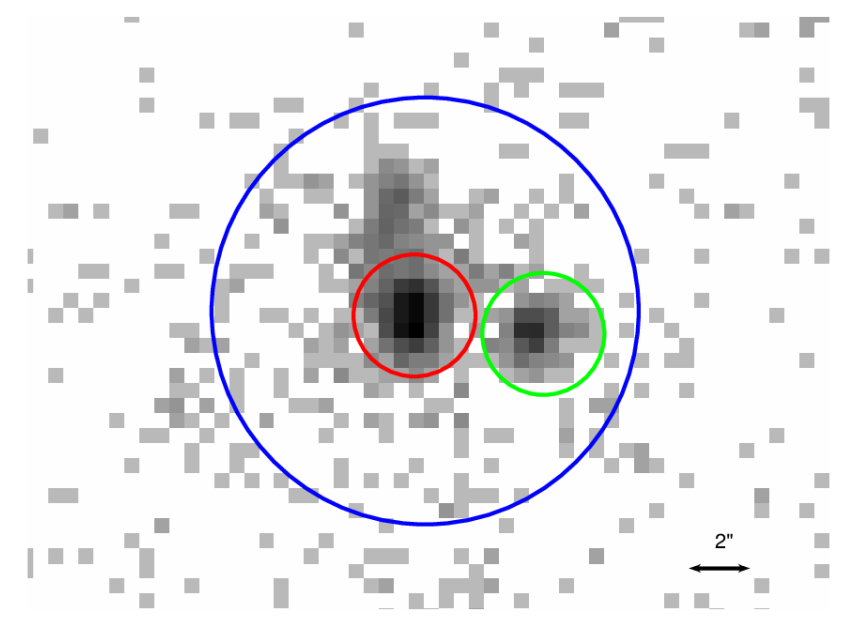

Figure 1. Chandra/ASIS-S image (0.4-8 keV) of the central region of Mrk 463. The 3 spectral extraction regions are shown by the circles for Mrk 463E (red), Mrk 463W (green), and the extended emission (blue).

was extracted from a circular region with a radius of 28 " centered at the middle point between the two galaxies, while the background was taken from a nearby circular region with a radius of 50". The RMF and ARF of each EPIC were generated by using the tasks RMFGEN and ARFGEN. The MOS1 and MOS2 spectra were combined into a single spectrum with ADDASCASPEC. The MOS1+MOS2 and PN spectra were binned to contain more than 30 counts per bin.

\section{X-RAY SPECTRAL ANALYSIS}

To best constrain the broadband spectral properties of Mrk 463, we simultaneously analyze six spectra: those of Mrk 463E, Mrk 463W, and the extended emission obtained with Chandra/ACIS-S $(0.4-7 \mathrm{keV}, 0.6-8 \mathrm{keV}$, $0.4-1.6 \mathrm{keV}$ ), and the total spectra (including all emission from Mrk 463) obtained with NuSTAR/FPMs (3$70 \mathrm{keV}), X M M-N e w t o n / E P I C-M O S s ~(0.47-11 \mathrm{keV})$, and $X M M-N e w t o n / E P I C-P N(0.49-12 \mathrm{keV})$, where the highest signal-to-noise ratios are achieved. The spectra folded with the energy responses are plotted in Figure 2 . We consider models independently for the three regions (Mrk 463E, Mrk 463W, and Extended) and apply each model to the corresponding Chandra spectrum, while the NuSTAR and XMM-Newton spectra are simultaneously fit with their summed model. Spectral fitting is performed on XSPEC (Arnaud 1996) v12.9.0 by adopting $\chi^{2}$-statistics.

In this analysis, we make a reasonable assumption that the absorption and the power-law photon index of Mrk 463E and Mrk 463W were constant among the three observations of NuSTAR, Chandra, XMM-Newton performed at different epochs. We take into account possible time variability of the AGN flux among the 3 epochs. Utilizing the fact that the absorptions of the two AGNs are different from each other (Bianchi et al. 2008) and that the low energy turnover observed in a few to $\sim 10 \mathrm{keV}$ is very sensitive to column densities of $N_{\mathrm{H}} \sim 10^{23}-10^{24} \mathrm{~cm}^{-2}$, it is feasible to spectroscopically separate their contributions in the NuSTAR and XMMNewton data even if they are spatially unresolved.

We take into account Galactic absorption in all spectral models, fixing the hydrogen column density to 1.98 $\times 10^{20} \mathrm{~cm}^{-2}$, as estimated from the HI map of the region (Kalberla et al. 2005). To correct for possible cross calibration uncertainties in absolute flux among the different instruments, we multiply an energy-independent constant factor (const0) to all the spectra by limiting its range to $0.8-1.2$. We set it to unity for the $N u S T A R$ and Chandra spectra as calibration reference.

\subsection{Model I}

We first apply analytical models for the three regions (Mrk 463E, Mrk 463W, and Extended). The spectrum of each nucleus consists of five components. In XSPEC terminology, it is expressed as:

$$
\begin{aligned}
& \text { Mrk } 463 \mathrm{E} / \mathrm{W}=\text { const } 0 * \text { phabs } \\
& *(\text { const } 1 * \text { zphabs } * \text { cabs } * \text { zpowerlw } * \text { zhighect } \\
&+ \text { const } 1 * \text { zphabs } * \text { pexmon } \\
&+ \text { const } 2 * \text { zpowerlw } * \text { zhighect } \\
&+ \text { apec }+ \text { zgauss }[\times 4 / 1])
\end{aligned}
$$

The const0 factor and phabs represent the crosscalibration constant and the Galactic absorption, respectively. The first term is the transmitted component from the AGN. It is modelled by an absorbed power law with an exponential cutoff, which is fixed at $360 \mathrm{keV}$ for consistency with the Ikeda et al. (2009) model (see below) $)^{1}$. The photon index and normalization are tied together among all the spectra. The const1 factor accounts for time variability between different epochs, which is fixed to unity for the $N u S T A R$ spectra but is left free to vary within $1 / 3$ and 3 for the Chandra and XMM-Newton spectra, consistently with the variability range observed from nearby AGNs over a timescale of $\sim 10$ years (Kawamuro et al. 2016). The cabs model takes into account the effect of Compton scattering in the line-of-sight, which is however negligible when the column density is $N_{\mathrm{H}}<10^{24} \mathrm{~cm}^{-2}$.

The second term represents a reflection component from cold matter in the circumnuclear region, to which we refer to as the "torus". We utilize the pexmon code (Nandra et al. 2007), which calculates a reflected continuum with $\mathrm{Fe}$ and $\mathrm{Ni}$ fluorescence lines from opticallythick cold matter. The photon index and the power-law normalization are linked to those of the transmitted com-

${ }^{1}$ We utilize the zhighect model whose e-folding and cutoff energies are fixed at $360 \mathrm{keV}$ and $0.01 \mathrm{keV}$, respectively. 
ponent. The reflection strength, $R \equiv \Omega / 2 \pi$ ( $\Omega$ is the solid angle of the reflector), is set to be free within a physically plausible range of $0<R \leq 2$. The inclination angle is fixed at $60^{\circ}$ as a representative value. We apply the same absorption to the reflection component as for the transmitted component, which provides a better fit $\left(\Delta \chi^{2}>2.46\right)$ than the case of no absorption. Assuming that the reflection flux follows the transmitted one with a time delay of $<$ a few years (i.e., its emitting region is located at $\lesssim 1$ pc from the SMBH; Minezaki \& Matsushita 2015. Gandhi et al. 2015), the same const1 factor as to the first component is multiplied to this component. Assuming a constant absolute flux for the reflection component, we found values of the parameters consistent within their $90 \%$ uncertainty with those obtained allowing the constant to vary.

The third term represents a scattered component from the AGN, modelled by an unabsorbed power law with an exponential cutoff at $360 \mathrm{keV}$. For convenience, we link the power-law normalizations at $1 \mathrm{keV}$ between the transmitted and scattered components and multiply a constant factor (const2) to the latter, which gives a scattering fraction, $f_{\text {scat }}{ }^{2}$. We do not link the photon indices of the transmitted and scattered components for Mrk 463E, whereas we link them for Mrk 463W because of the limited photon statistics of its Chandra spectrum. We assume that this component comes from regions larger than a few pc scale and thus that absolute flux is constant over the 3 epochs.

The fourth term represents optically-thin thermal emission modeled by the apec code (Smith et al. 2001), which is expected if star forming activity is present in the host galaxy (see Section 4). The fifth term represents additional emission line features modeled by zgauss that cannot be explained by the lines included in the above apec component; we consider that they are most likely recombination lines and radiative recombination continuum (RRC) from a photoionized gas irradiated by the AGN (e.g., Bianchi et al. 2006). We select them from the list of narrow lines summarized in Table 1 of Bianchi et al. (2008) for each galaxy, and finally add them to the model if they are found to significantly improve the fit with $\Delta \chi^{2}>2.71$ (i.e., at a $>90 \%$ confidence limit). Here we also consider emission lines only detected with $X M M-N e w t o n$, assuming that they originate predominantly from Mrk 463E, which is brighter than Mrk 463W and the extended emission. We finally adopt lines at $0.654 \mathrm{keV}(\mathrm{O}$ VIII $\mathrm{K} \alpha), 0.739 \mathrm{keV}$ (O VII RRC), $1.342 \mathrm{keV}(\mathrm{Mg}$ XI K $\alpha$ ), and $4.070 \mathrm{keV}$ ( Ca XX K $\alpha$ ) for Mrk 463E, and at $1.022 \mathrm{keV}(\mathrm{Ne} \mathrm{X} \mathrm{K} \alpha)$ for Mrk 463W. We assume that the fluxes of the apec and zgauss components were constant over the 3 epochs.

The soft X-ray extended emission consists of three components, expressed as:

$$
\begin{aligned}
& \text { Extended }=\text { const } 0 * \text { phabs } \\
& \quad *(\text { zpowerlw } * \text { zhighect }+ \text { apec }+ \text { zgauss }) .
\end{aligned}
$$

The first term represents a scattered component from the AGN (Mrk 463E and/or Mrk 463W). The second term

2 We note that contribution from high mass X-ray binaries (HMXBs) in the host galaxy may be included in this unabsorbed power law component. represents optically-thin thermal emission (apec). The third term is an additional emission line at $1.022 \mathrm{keV}$ $(\mathrm{Ne} \mathrm{X} \mathrm{K} \alpha)$, which is selected in the same way as for the Mrk 463E/W spectra. We assume that the fluxes of all these components were constant over the 3 epochs.

Model I is found to well reproduce the overall spectra of Mrk $463\left(\chi^{2} /\right.$ d.o.f $=239.9 / 215$ for the total 6 spectra). The folded best-fit models are overplotted in the left panels of Figure 2, and the unfolded best-fit models for Mrk 463E and Mrk 463W are plotted in the left panels of Figure 3 . The best-fit parameters are summarized in Table 2 along with the intrinsic AGN luminosities. The hydrogen column density of Mrk 463E and Mrk $463 \mathrm{~W}$ are estimated to be $N_{\mathrm{H}}=5.8_{-1.4}^{+2.7} \times 10^{23} \mathrm{~cm}^{-2}$ and $N_{\mathrm{H}}=2.5 \pm 0.6 \times 10^{23} \mathrm{~cm}^{-2}$, respectively. The reflection components from the tori are significantly detected from both galaxies.

\subsection{Model II}

As a more physically self-consistent model, we next apply the "e-torus" model (Ikeda et al. 2009) to the AGN spectra (Model II). The e-torus model is a Monte-Carlo based spectral model from a uniform-density torus of nearly spherical geometry with bipolar cone holes. The torus parameters are the ratio of the inner to outer radii (fixed at 0.01), the half opening angle of the torus, the inclination angle, and the column density along the equatorial plane. As the incident spectrum, a power law with a photon index between 1.5-2.5 and an exponential cutoff at $360 \mathrm{keV}$ is assumed. The reflected continuum and iron-K fluorescence lines from the torus are provided as a table model in XSPEC.

The spectrum of each nucleus is expressed in XSPEC terminology as:

$$
\begin{aligned}
& \text { Mrk } 463 \mathrm{E} / \mathrm{W}=\text { const } 0 * \text { phabs } \\
& *(\text { const } 1 * \text { torusabs } * \text { zpowerlw } * \text { zhighect } \\
& + \text { const } 1 * \text { zpowerlw } * \text { zhighect } \\
& \quad * \text { mtable }\{\text { refl_all_20161115M.fits }\} \\
& \quad+\text { const } 1 * \text { atable }\{\text { refl_fe_torus.fits }\} \\
& + \text { const } 2 * \text { zpowerlw } * \text { zhighect } \\
& + \text { apec }+ \text { zgauss }[\times 4 / 1])
\end{aligned}
$$

The model consists of five components: (1) a transmitted component from the AGN, whose column density along the equatorial plane is self-consistently calculated from the torus geometry by torusabs (Eguchi et al. 2011), (2) a reflection component from the torus including an iron-K $\alpha$ emission line $(6.4 \mathrm{keV})$, (3) a scattered component from the AGN, (4) optically-thin thermal emission in the host galaxy, and (5) additional emission lines required from the data. The half opening angle and the inclination angle of the torus are fixed at $60^{\circ}$ and $80^{\circ}$ respectively, since they are difficult to constrain with our spectra, due to the limited photon statistics. We have confirmed that the choice of these parameters does not affect significantly our results.

\footnotetext{
${ }^{3}$ In this case, the ratio of the line-of-sight column density $\left(N_{\mathrm{H}}^{\mathrm{LS}}\right.$ to the column density along the equatorial plane $\left(N_{\mathrm{H}}^{\mathrm{Eq}}\right)$ is 0.995 (Ikeda et al. 2009).
} 

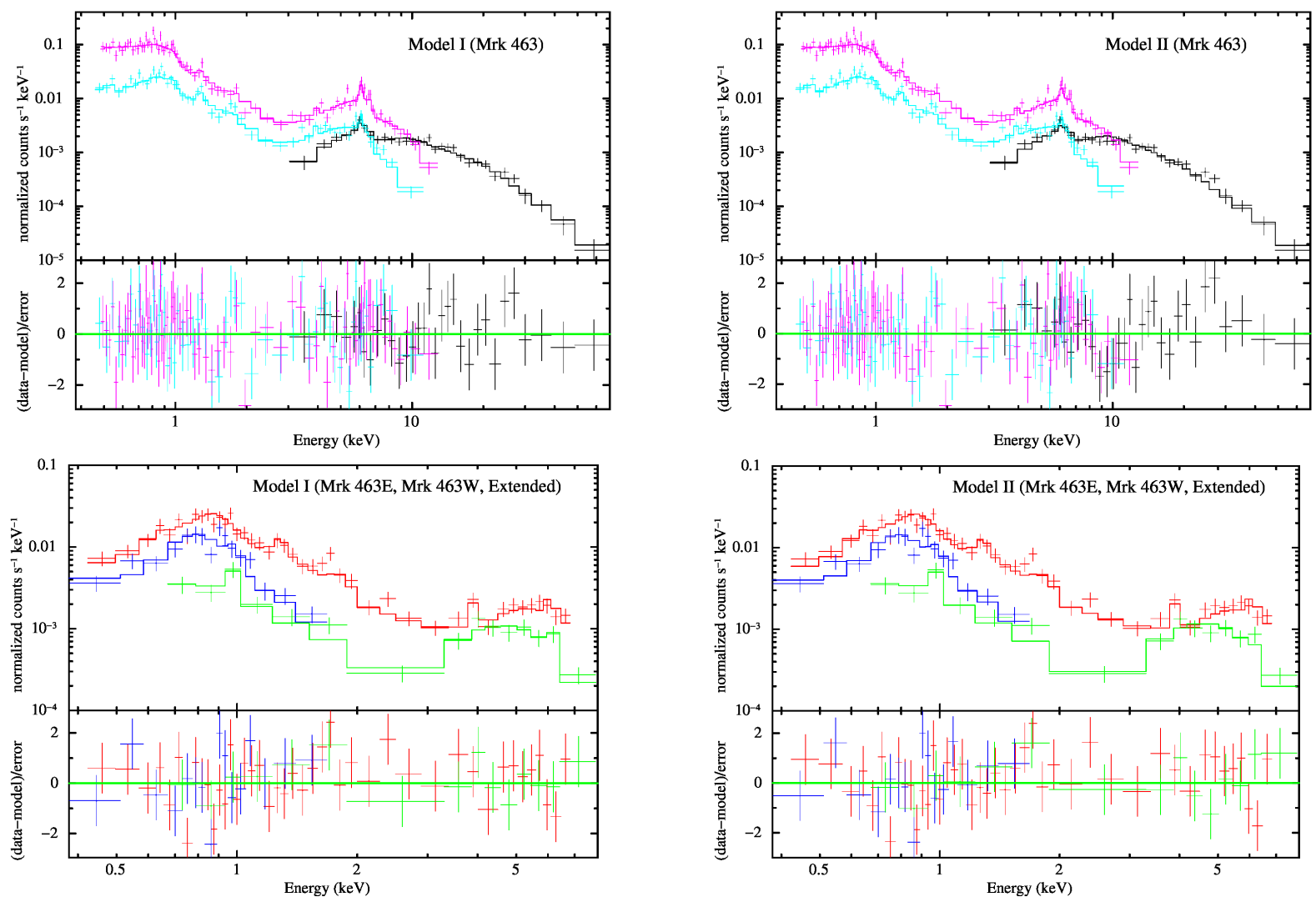

Figure 2. The observed spectra of Mrk 463 folded with the energy responses. The best-fit models are overplotted. Left: Model I. Right: Model II. Upper: The black, light blue, and magenta crosses represent the NuSTAR, XMM-Newton/MOS, and XMM-Newton/PN data of the total spectrum of Mrk 463, respectively. Lower: The red, green, and blue crosses represent the Chandra spectra of Mrk 463E, Mrk $463 \mathrm{~W}$, and the extended emission, respectively. The residuals in units of $\sigma$ are plotted in the lower panels.

The spectrum of the soft X-ray extended emission is the same we used for Model I:

$$
\begin{aligned}
& \text { Extended }=\text { const } 0 * \text { phabs } \\
& \quad *(\text { zpowerlw } * \text { zhighect }+ \text { apec }+ \text { zgauss }) .
\end{aligned}
$$

Model II also gives a good fit $\left(\chi^{2} /\right.$ d.o.f. $\left.=257.9 / 217\right)$ of the overall spectra of Mrk 463. The best-fit models are plotted in the right panels of Figure 2 (folded spectra) and in the right panels of Figure 3 (unfolded spectra). The best-fit parameters and intrinsic AGN luminosities are summarized in Table 2. The line-of-sight hydrogen column densities of Mrk 463E and Mrk 463W are estimated to be $N_{\mathrm{H}}=7.5_{-1.2}^{+1.3} \times 10^{23} \mathrm{~cm}^{-2}$ and $N_{\mathrm{H}}=2.9_{-0.5}^{+0.6} \times 10^{23} \mathrm{~cm}^{-2}$, respectively.

\section{DISCUSSION}

We have presented our broadband X-ray $(0.4-70 \mathrm{keV})$ spectral analysis of Mrk 463 by utilizing all available data of NuSTAR, Chandra, and XMM-Newton observations, carried out in 2014, 2004, and 2001, respectively. With a help of the Chandra data, we are able to separate broadband spectra of Mrk 463E, Mrk 463W, and of the extended emission, by assuming that their spectral shapes (not fluxes) were constant among these epochs. In this section, we discuss our results, focusing on the two nuclei, Mrk 463E and Mrk 463W.

We have shown that both the analytical model (Model I) and the numerical torus model (Model II) well reproduce the spectra of Mrk 463E and Mrk 463W.
Bianchi et al. (2008) adopted an empirical model consisting of an absorbed power-law, unabsorbed powerlaw, and individual emission lines, whereas we include a reflection component from the torus and an opticallythin thermal emission component as physically motivated models. Although it is difficult to disentangle origins in a collisional gas or a photoionized gas for the soft Xray emission using the $\mathrm{CCD}$ data alone, we verify that the derived soft X-ray luminosities of the thermal components are reasonable as compared with the star formation rate of the galaxies. From the 42.5-122.5 $\mu \mathrm{m}$ luminosity $\left(5.7 \times 10^{44} \mathrm{erg} \mathrm{s}^{-1}\right.$ as estimate from the IRAS $60 \mu \mathrm{m}$ and $100 \mu \mathrm{m}$ photometries) and the 8-1000 $\mu \mathrm{m}$ luminosity $\left(2.4 \times 10^{45} \mathrm{erg} \mathrm{s}^{-1}\right.$, Imanishi \& Saito 2014), of Mrk 463 , the $0.5-2 \mathrm{keV}$ luminosity originating from the star formation activity is expected to be $1.3 \times 10^{41} \mathrm{erg} \mathrm{s}^{-1}$ and $7 \times 10^{40} \mathrm{erg} \mathrm{s}^{-1}$ (mean values), based on the relations of Ranalli et al. (2003) and Iwasawa et al. (2011a), respectively. The total observed $0.5-2 \mathrm{keV}$ luminosity of the apec components in Mrk $463\left(2.9 \times 10^{41} \mathrm{erg} \mathrm{s}^{-1}\right)$ is consistent with these predictions within the scatter. With Model I, where the pexmon code is utilized, we detect significant reflection components from both galaxies, as expected from the presence of strong iron- $\mathrm{K} \alpha$ emission lines in the Chandra and XMM-Newton spectra. These reflection features are consistently explained by the numerical torus model (Model II). We confirm that the basic spectral parameters obtained with Model I and Model II are consistent with each other. Hereafter, we refer to the results obtained with Model II unless otherwise stated. 
Table 2

Best-fit Spectral Parameters

\begin{tabular}{|c|c|c|c|c|c|}
\hline Region & No. & Parameter & Model I & Model II & Units \\
\hline \multirow[t]{16}{*}{ Mrk 463E } & $(1)$ & $N_{\mathrm{H}}$ & $5.8_{-1.4}^{+2.7}$ & $7.5_{-1.2}^{+1.3}$ & $10^{23} \mathrm{~cm}^{-2}$ \\
\hline & $(2)$ & $\Gamma_{\mathrm{AGN}}$ & $1.5_{-0.4}^{+1.1}$ & $1.50_{-0.00(*)}^{+0.14}$ & \\
\hline & $(3)$ & $A_{\mathrm{AGN}}$ & $2.9_{-2.2}^{+5.8}$ & $5.5_{-0.8}^{+3.0}$ & $10^{-4} \mathrm{keV}^{-1} \mathrm{~cm}^{-2} \mathrm{~s}^{-1}$ \\
\hline & $(4)$ & $R$ & $1.01_{-0.86}^{+0.84}$ & $\cdots$ & \\
\hline & $(5)$ & $f_{\text {scat }}$ & $5.5_{-4.9}^{+18.8}$ & $2.7_{-1.1}^{+0.8}$ & $\%$ \\
\hline & $(6)$ & $\Gamma_{\text {scat }}$ & $1.47_{-0.26}^{+0.22}$ & $1.38_{-0.27}^{+0.22}$ & \\
\hline & $(7)$ & $k \mathrm{~T}$ & $0.89_{-0.10}^{+0.07}$ & $0.89_{-0.10}^{+0.07}$ & $\mathrm{keV}$ \\
\hline & $(8)$ & $A_{\text {apec }}$ & $0.96_{-0.19}^{+0.21}$ & $1.00_{-0.19}^{+0.21}$ & $10^{-5} \mathrm{~cm}^{-5}$ \\
\hline & $(9)$ & $A(0.654 \mathrm{keV})$ & $2.5 \pm 1.9$ & $3.0 \pm 1.9$ & $10^{-6}$ \\
\hline & $(10)$ & $A(0.739 \mathrm{keV})$ & $0.9_{-0.9(*)}^{+1.6}$ & $0.9_{-0.9(*)}^{+1.6}$ & $10^{-6}$ \\
\hline & $(11)$ & $A(1.342 \mathrm{keV})$ & $1.0 \pm 0.5$ & $1.0 \pm 0.5$ & $10^{-6}$ \\
\hline & $(12)$ & $A(4.070 \mathrm{keV})$ & $0.7 \pm 0.4$ & $0.8 \pm 0.4$ & $10^{-6}$ \\
\hline & (13) & $N_{\text {Chandra }}$ & $1.7_{-0.8}^{+1.3(*)}$ & $1.33_{-0.35}^{+0.45}$ & \\
\hline & $(14)$ & $N_{\mathrm{XMM}}$ & $1.6_{-0.5}^{+1.4(*)}$ & $1.26_{-0.26}^{+0.31}$ & \\
\hline & $(15)$ & $F_{2-10 \mathrm{keV}}$ & $3.11 \pm 0.11$ & $3.98 \pm 0.13$ & $10^{-13} \mathrm{erg} \mathrm{cm}^{-2} \mathrm{~s}^{-1}$ \\
\hline & $(16)$ & $L_{2-10 \mathrm{keV}}$ & $0.85_{-0.29}^{+0.41}$ & $1.64 \pm 0.21$ & $10^{43} \mathrm{erg} \mathrm{s}^{-1}$ \\
\hline \multirow[t]{12}{*}{ Mrk 463W } & $(17)$ & $N_{\mathrm{H}}$ & $2.5 \pm 0.6$ & $2.9_{-0.5}^{+0.6}$ & $10^{23} \mathrm{~cm}^{-2}$ \\
\hline & $(18)$ & $\Gamma_{\mathrm{AGN}}$ & $2.1_{-0.7}^{+1.0}$ & $2.42_{-0.45}^{+0.08(*)}$ & \\
\hline & (19) & $A_{\mathrm{AGN}}$ & $4.9_{-3.7}^{+18}$ & $8.2_{-5.3}^{+5.7}$ & $10^{-4} \mathrm{keV}^{-1} \mathrm{~cm}^{-2} \mathrm{~s}^{-1}$ \\
\hline & $(20)$ & $R$ & $2.0_{-1.2}^{+0.0(*)}$ & $\cdots$ & \\
\hline & $(21)$ & $f_{\text {scat }}$ & $1.0_{-0.6}^{+1.6}$ & $0.7_{-0.3}^{+0.9}$ & $\%$ \\
\hline & $(22)$ & $k \mathrm{~T}$ & $0.16_{-0.12}^{+0.04}$ & $0.15_{-0.11}^{+0.04}$ & $\mathrm{keV}$ \\
\hline & $(23)$ & $A_{\text {apec }^{\dagger}}$ & $1.30_{-0.57}^{-0.12}$ & $1.50_{-0.76}^{-0.11}$ & $10^{-5} \mathrm{~cm}^{-5}$ \\
\hline & $(24)$ & $A(1.022 \mathrm{keV})$ & $1.0 \pm 0.6$ & $0.9 \pm 0.6$ & $10^{-6}$ \\
\hline & $(25)$ & $N_{\text {Chandra }}$ & $0.64_{-0.20}^{+0.50}$ & $0.82_{-0.24}^{+0.65}$ & \\
\hline & $(26)$ & $N_{\mathrm{XMM}}$ & $0.60_{-0.27(*)}^{+0.39}$ & $0.79_{-0.26}^{+0.51}$ & \\
\hline & $(27)$ & $F_{2-10 \mathrm{keV}}$ & $3.24 \pm 0.23$ & $2.31 \pm 0.17$ & $10^{-13} \mathrm{erg} \mathrm{cm}^{-2} \mathrm{~s}^{-1}$ \\
\hline & $(28)$ & $L_{2-10 \mathrm{keV}}$ & $0.56_{-0.20}^{+0.22}$ & $0.62_{-0.28}^{+0.29}$ & $10^{43} \mathrm{erg} \mathrm{s}^{-1}$ \\
\hline \multirow[t]{10}{*}{ Extended } & $(29)$ & $\Gamma_{\text {Ext }}$ & $2.58_{-0.44}^{+0.48}$ & $2.50_{-0.36}^{+0.00(*)}$ & \\
\hline & $(30)$ & $A_{\mathrm{Ext}}$ & $5.1 \pm 1.5$ & $5.2 \pm 1.4$ & $10^{-6} \mathrm{keV}^{-1} \mathrm{~cm}^{-2} \mathrm{~s}^{-1}$ \\
\hline & (31) & $k \mathrm{~T}$ & $0.66_{-0.08}^{+0.12}$ & $0.65_{-0.08}^{+0.11}$ & $\mathrm{keV}$ \\
\hline & $(32)$ & $A_{\text {apec }}$ & $0.53 \pm 0.10$ & $0.53 \pm 0.10$ & $10^{-5} \mathrm{~cm}^{-5}$ \\
\hline & (33) & $A(1.022 \mathrm{keV})$ & $1.1 \pm 0.7$ & $1.2 \pm 0.7$ & $10^{-6}$ \\
\hline & $(34)$ & $F_{0.5-2 \mathrm{keV}}$ & $2.43 \pm 0.19$ & $2.45 \pm 0.19$ & $10^{-14} \mathrm{erg} \mathrm{cm}^{-2} \mathrm{~s}^{-1}$ \\
\hline & $(35)$ & $L_{0.5-2 \mathrm{keV}}$ & $1.49 \pm 0.11$ & $1.49 \pm 0.11$ & $10^{41} \mathrm{erg} \mathrm{s}^{-1}$ \\
\hline & $(36)$ & $C_{\mathrm{XMM}-\mathrm{MOS}}$ & $1.15_{-0.08}^{+0.05(*)}$ & $1.15_{-0.07}^{+0.05(*)}$ & \\
\hline & $(37)$ & $C_{\mathrm{XMM}-\mathrm{PN}}$ & $1.10 \pm 0.07$ & $1.09 \pm 0.07$ & \\
\hline & & $\chi^{2} /$ d.o.f. $\left(\chi_{r}^{2}\right)$ & $239.9 / 215(1.12)$ & $257.9 / 217(1.19)$ & \\
\hline
\end{tabular}

Note. - (1) Hydrogen column density. (2) Power law photon index of the transmitted component. (3) Power law normalization at 1 keV. (4) Reflection strength $(R=\Omega / 2 \pi)$ in the pexmon model. (5) Scattering fraction. (6) Power law photon index of the scattering component. (7) Temperature of the apec model. (8) Normalization of the apec model. (9) Normalization of the O VIII K $\alpha$. (10) Normalization of the O VII RRC. (11) Normalization of the Mg XI K $\alpha$ line. (12) Normalization of the Ca XX K $\alpha$ line. (13) Time variability constant of Chandra relative to NuSTAR. (14) Time variability constant of XMM-Newton relative to NuSTAR. (15) Observed flux in the 2-10 keV band (at the NuSTAR observation). The error is estimated by fixing all the spectral parameters at the best-fit values except the overall normalizations for the three regions. (16) Intrinsic (de-absorbed) AGN luminosity in the rest frame 2-10 keV band (at the NuSTAR observation). The error is estimated by fixing the photon index at the best-fit value. (17)-(23) Parameters for Mrk $463 \mathrm{~W}$ corresponding to (1)-(5) and (7)-(8), respectively. (24) Normalization of the Ne X K $\alpha$ line. (25)-(28) Parameters for Mrk 463W corresponding to (13)-(16), respectively. (29) Power-law photon index. (30) Power-law normalization $1 \mathrm{keV}$. (31) Temperature of the apec model. (32) Normalization of the apec model. (33) Normalization of the Ne X K $\alpha$ line. (34) Observed flux of the extended emission in the 0.5-2 keV band. (35) Luminosity of the extended emission in the rest-frame $0.5-2 \mathrm{keV}$ band. The same relative error as in the observed flux is attached. (36) Cross-normalization of $X M M$ /MOSs relative to NUSTAR. (37) Cross-normalization of XMM/PN relative to NuSTAR.

$\dagger$ The upper limit cannot be well constrained owing to the lack of data below $0.7 \mathrm{keV}$ in the Chandra Mrk $463 \mathrm{~W}$ spectrum.

* The parameter reaches a limit of its allowed range. 

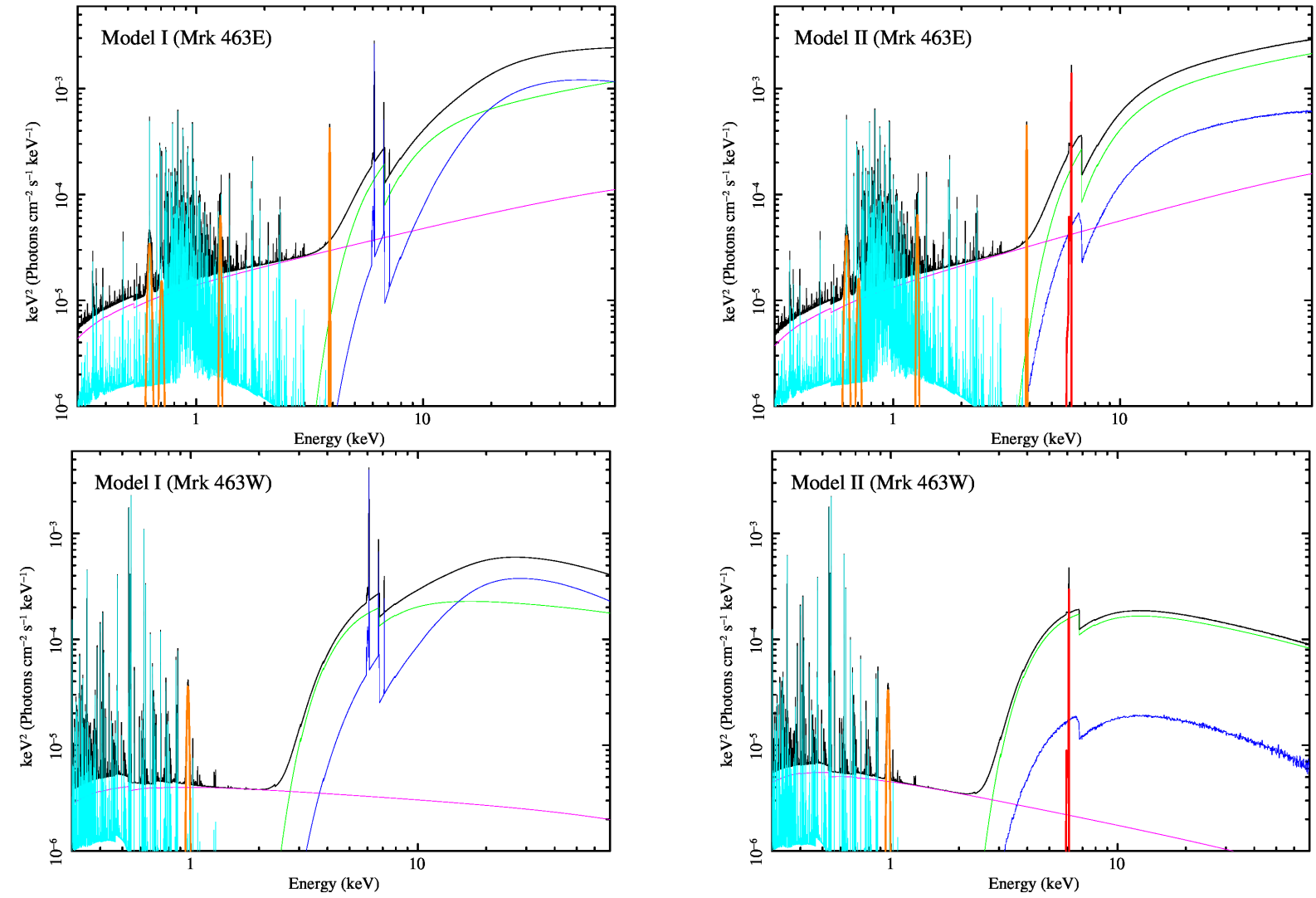

Figure 3. The best-fit models in units of $E I_{E}$ (where $I_{E}$ is the energy flux at the energy $E$ ). Left: Model I. Right: Model II. Upper: Mrk 463E. Lower: Mrk 463W. The black, green, magenta, blue, red, cyan, and orange lines represent the total model, transmitted component, scattered component, reflection component, iron $\mathrm{K} \alpha$ emission line, optically-thin thermal emission, and additional emission lines, respectively. 


\subsection{Mrk $463 E$}

We have determined the line-of-sight column density of Mrk $463 \mathrm{E}$ to be $7.5 \times 10^{23} \mathrm{~cm}^{-2}$, which is consistent with the result by Bianchi et al. (2008), and thus confirm that the AGN is heavily obscured but not Compton-thick. We obtain a flatter slope $(\Gamma \simeq 1.5)$ for the AGN transmitted component than Bianchi et al. (2008) $(\Gamma \simeq 2.3)$. This is most likely because an optically-thin thermal component was not included in their model, leading to a steeper slope of the unabsorbed power law component linked to that of the transmitted component. Our analysis suggests that the fluxes of Mrk $463 \mathrm{E}$ in the $N u S$ TAR observation in 2014 were $N_{\text {Chandra }} \approx 1.33$ and $N_{\mathrm{XMM}} \approx 1.26$ times lower than those in the Chandra (2004) and XMM-Newton (2001) observations, respectively. The best-fit intrinsic (de-absorbed) $2-10 \mathrm{keV} \mathrm{lu}-$ minosities range from $1.6 \times 10^{43} \mathrm{erg} \mathrm{s}^{-1}$ (2014) to 2.2 $\times 10^{43} \mathrm{erg} \mathrm{s}^{-1}$ (2004), confirming that Mrk 463E has a Seyfert class AGN. In the following discussions centered on the X-ray luminosity, we take into account the range of this variability.

Comparison of luminosities between X-rays and highexcitation mid-IR lines from the narrow line region (NLR), such as [O IV] $25.89 \mu \mathrm{m}$ and [Ne V] $14.32 \mu \mathrm{m}$, gives useful insight into the spectral energy distribution (SED) and circumnuclear structure of the AGN. Since such mid-IR lines emitted from the NLR are less attenuated by dust in the host galaxy and are less contaminated by star-formation activities, they are generally thought to be better indicators of the intrinsic AGN luminosity than the widely used [O III] $\lambda 5007$ line (Heckman et al. 2005). Since these MIR lines are produced by irradiation of UV photons from the AGN, their luminosity ratios to $\mathrm{X}$-rays reflect the intrinsic AGN SED in the UV to X-ray band, unless the nucleus is deeply "buried" by dust (i.e., the obscurer has a large covering factor) and hence the NLR is underdeveloped (Oda et al. 2017).

Figure 4 plots the relation between the de-absorbed 2 $10 \mathrm{keV}$ luminosity and the [O IV] $25.89 \mu \mathrm{m}$ luminosity for Mrk 463E (Armus et al. 2004). As noticed, the luminosity ratio between X-ray (2-10 keV) and [O IV] $25.89 \mu \mathrm{m}$ of Mrk $463 \mathrm{E}$ is smaller than the averaged values of normal Seyfert galaxies by a factor of $\sim 5$, indicating that it is "X-ray weak" relative to the UV luminosity. The X-ray underluminosity was also implied from the broad optical/NIR emission lines (Imanishi \& Terashima 2004) and from the [O III] $\lambda 5007$ line (Bianchi et al. 2008). 'The $\mathrm{X}$-ray faint SED (i.e., a large bolometric to X-ray luminosity ratio) implies that the AGN has a high Eddington ratio (Vasudevan \& Fabian 2007), that is, contain a rapidly growing SMBH. We also note that this result provides no evidence that the nucleus is deeply "buried" by dust, because it would lead to the opposite trend (i.e., brighter in the X-rays than in [O IV]). For reference, we also plot the data of IRAS 05189-2524, IRAS 13120-5453, Mrk 273, and UGC 5101 (Veilleux et al. 2009, Teng et al. 2015. Oda et al. 2017), which are ULIRGs observed with NuSTAR. We note that Mrk 273, a late-stage merging galaxy with one or two obscured AGNs (Iwasawa et al. 2011b), may be a similar system to Mrk 463, whereas the other ULIRGs showing same X-ray vs [O IV] relations as normal Seyferts may contain "buried" AGNs with high Eddington ratios, where the [O IV] emission is signifi-

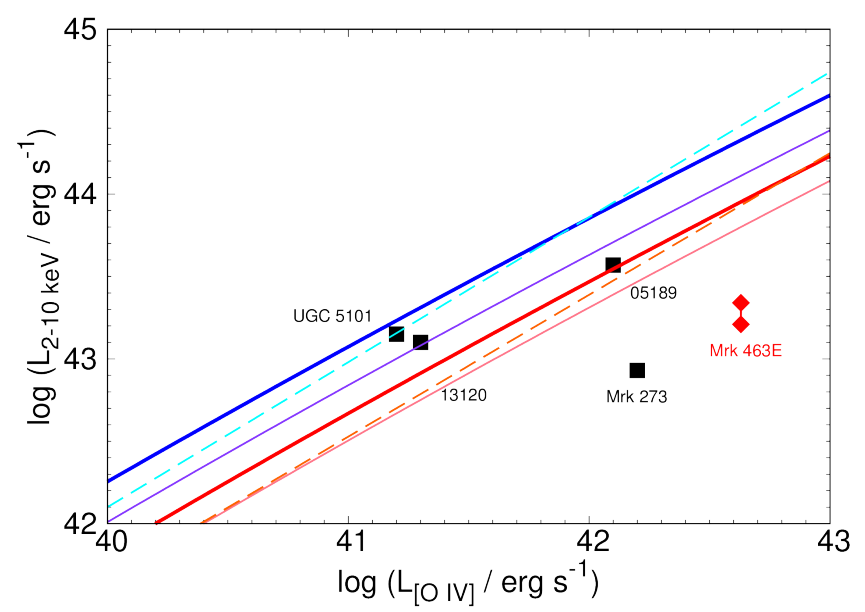

Figure 4. The $2-10 \mathrm{keV}$ versus [O IV] $25.89 \mu \mathrm{m}$ luminosity plot of local U/LIRGs. Mrk 463E is marked with the red diamonds referring to the X-ray luminosities in 2014 (lower) and 2004 (upper). The Compton-thick ULIRGs in Teng et al. (2015) and UGC 5101 in (Oda et al. 2017) are plotted with the black squares. The bold blue/red curves and solid purple/pink lines represent the averaged relations for local Seyfert 1s/2s and Compton-thin/thick Seyfert 2s (Rigby et al. 2009), respectively. The dashed cyan/orange lines show the Seyfert 1s/2s relations obtained by Liu et al. (2014).

cantly suppressed (Oda et al. 2017).

We quantitatively estimate the Eddington ratio of Mrk 463E, using $L^{\prime}$-band $(3.8 \mu \mathrm{m})$, [O IV] $25.89 \mu \mathrm{m}$, and $[\mathrm{Ne} \mathrm{V}] 14.32 \mu \mathrm{m}$ luminosities. The $L^{\prime}$-band $(3.8 \mu \mathrm{m})$ emission is produced by AGN-heated hot dust in a vicinity ( a few pc) of an AGN (Barvainis 1987), and hence its luminosity (after subtracting a starburst contribution) is also a good indicator of the AGN bolometric luminosity. By comparing the $K$-band $(2.2 \mu \mathrm{m})$ and $L^{\prime}$ band luminosities, Imanishi \& Saito (2014) estimated the AGN contribution to the $L^{\prime}$-band luminosity of Mrk $463 \mathrm{E}$ is $\sim 100 \%$. Table 3 summarizes the luminosities in the $L^{\prime}$ band $(3.8 \mu \mathrm{m})$, [O IV] $25.89 \mu \mathrm{m}$, and [Ne V] $14.32 \mu \mathrm{m}$, as well as the corresponding bolometric luminosities based on the relations by Risaliti et al. (2010), Rigby et al. (2009), and Satyapal et al. (2007), respectively. These bolometric correction factors depend on the torus opening angle; here we assume that those of Mrk 463E is similar to the averaged values of normal Seyfert galaxies because its nucleus is unlikely to be deeply buried (see above). We find these bolometric luminosities agree with one another within a factor of 2 . They are also similar to the total infrared luminosity of Mrk 463 (sum of Mrk 463E and Mrk 463W), indicating that Mrk 463E is an AGN-dominated LIRG. Adopting the black hole mass of Mrk 463E, $5.5 \times 10^{7} M_{\odot}$, as determined by Dasyra et al. (2011) using the $M_{\mathrm{BH}}-\sigma_{*}$ relation, we calculate the Eddington ratio $\left(\lambda_{\mathrm{Edd}}\right)$ for each luminosity estimate (Table 3 ). We find $\lambda_{\text {Edd }}=0.4-0.8$, thus confirming that the AGN in Mrk 463E has a high Eddington ratio. We also confirm that the X-ray to bolometric luminosity ratios is large, $\kappa_{2-10 \mathrm{keV}} \equiv L_{\mathrm{bol}} / L_{2-10 \mathrm{keV}} \sim 110-410$ (Table 3).

We note that AGN time variability (i.e., the X-ray observations were performed by chance when the AGN activity was largely suppressed) is unlikely to be a major origin of the X-ray weakness. The $L^{\prime}$-band emission predominantly comes from AGN-heated dust of $\sim 1000 \mathrm{~K}$, whose sublimation radius is estimated to be $\approx 2-3 \mathrm{pc}$ for 
Table 3

Summary of Bolometric Luminosity and Eddington Ratio of Mrk 463E

\begin{tabular}{|c|c|c|c|c|}
\hline $\begin{array}{l}\text { Method } \\
(1)\end{array}$ & $\begin{array}{c}L_{\mathrm{obs}} \\
(2)\end{array}$ & $\begin{array}{c}\begin{array}{c}\mathrm{bGN} \\
(3)\end{array} \\
\mathrm{AGO}\end{array}$ & $\begin{array}{c}\kappa_{2-10 \mathrm{keV}} \\
(4)\end{array}$ & $\begin{array}{c}\lambda_{\mathrm{Edd}} \\
(5)\end{array}$ \\
\hline$L^{\prime}$-band $(3.8 \mu \mathrm{m})$ & 550 & 2.75 & $110-190$ & 0.4 \\
\hline$[\mathrm{O}$ IV] $(25.89 \mu \mathrm{m})$ & 4.27 & 5.63 & $230-390$ & 0.8 \\
\hline$[\mathrm{Ne} \mathrm{V}](14.32 \mu \mathrm{m})$ & 1.15 & 5.88 & $240-410$ & 0.8 \\
\hline
\end{tabular}

Note. - (1) AGN bolometric luminosity indicator. (2) Its observed luminosity in units of $10^{42} \mathrm{erg} \mathrm{s}^{-1}$. (3) Estimated AGN bolometric luminosity in units of $10^{45} \mathrm{erg} \mathrm{s}^{-1}$. (4) X-ray $(2-10 \mathrm{keV})$ to bolometric correction factor. The statistical error in the X-ray luminosity and time variability range based on Model II are taken into account. (5) Eddington ratio calculated with the SMBH mass by Dasyra et al. (2011).

$L_{\text {bol }}=(3-6) \times 10^{45} \mathrm{erg} \mathrm{s}^{-1}$ (Barvainis 1987). Thus, the $L^{\prime}$-band luminosity of this object should reflect its past AGN activity over several to ten years. Since the $L^{\prime}$-band data of Mrk 463E were taken in 2012 (Imanishi \& Saito 2014), they can be reasonably compared with the X-ray luminosities measured in 2004 (with Chandra) and 2001 (with XMM-Newton), on which our discussion is based.

To summarize, we confirm that the AGN in Mrk 463E has a high Eddington rate (i.e., the $\mathrm{SMBH}$ is rapidly growing) and is X-ray underluminous compared with normal Seyferts. These properties are consistent with the general trend between $\lambda_{\text {Edd }}$ and $\kappa_{2-10 \mathrm{keV}}$ (Vasudevan \& Fabian 2007). We infer that the merger triggered efficient gas fueling onto the SMBH. Unlike typical ULIRGs, however, the nucleus of Mrk 463E is not deeply buried by dust. This is in line with the evolutionary scenario of mergers in which obscuration of the nuclei by galactic-scale gas/dust becomes more significant toward later stages (Ricci et al. 2017).

\subsection{Mrk $463 W$}

We determine the absorption of Mrk $463 \mathrm{~W}$ to be $2.9 \times 10^{23} \mathrm{~cm}^{-2}$, confirming that the galaxy contains a Compton-thin obscured AGN. The column density and photon index $(\Gamma \simeq 2.4)$ are consistent with the results by Bianchi et al. (2008). Our analysis suggests that the flux of Mrk 463E in 2014 was higher than those in 2004 and 2001 by factors of $N_{\text {Chandra }} \approx 0.82$ and $N_{\mathrm{XMM}} \approx 0.79$, respectively, although this variation is not statistically significant. The best-fit intrinsic $2-10 \mathrm{keV}$ luminosities range from $6.2 \times 10^{42} \mathrm{erg} \mathrm{s}^{-1}(2014)$ to $5.1 \times 10^{42} \mathrm{erg} \mathrm{s}^{-1}$ (2004). Below we discuss our results by comparison with the $L^{\prime}$-band luminosity. Unfortunately, neither the [O IV] $25.89 \mu \mathrm{m}$ and [Ne V] $14.32 \mu \mathrm{m}$ luminosities nor the black hole mass are not available for Mrk 463W.

We obtain a bolometric luminosity of Mrk 463W of $8.5 \times 10^{42}$ erg $\mathrm{s}^{-1}$, using the $L^{\prime}$-band luminosity after subtracting the $68 \%$ starburst contribution (Risaliti et al. 2010; Imanishi \& Saito 2014). This yields an unusually small bolometric to $2-10 \mathrm{keV}$ luminosity ratio, $\kappa_{2-10 \mathrm{keV}} \sim 1-3$, as compared with typical AGNs $\left(\kappa_{2-10 \mathrm{keV}} \sim 10-70 ;\right.$ Vasudevan \& Fabian 2007). We consider three possibilities to explain this very small value. The first possibility is that the AGN torus in Mrk 463W has a very low covering factor, to which the mid-IR luminosity is approximately proportional. We infer it unlikely because this contradicts with the strong reflection component $(R>0.8)$ seen in the X-ray spectrum. The second one is time variability. When an AGN is rapidly brightening from a quiescent state, it becomes bright in the UV/X-ray band first before the radiation reaches to circumnuclear dust that emit mid-IR radiation. The fact that Mrk $463 \mathrm{~W}$ was already X-ray bright in 2001 but faint in the $L^{\prime}$-band in 2012 (Imanishi \& Saito 2014) suggests that the $L^{\prime}$-band emission would be mainly produced at distances of $>3$ pc. These distances are, however, much larger than the sublimation radius of dust with $\sim 1000 \mathrm{~K}$ for $L_{\mathrm{bol}} \sim 10^{44} \mathrm{erg} \mathrm{s}^{-1}$ (converted from the X-ray luminosity with $\kappa_{2-10 \mathrm{keV}} \sim 20$ by assuming a normal SED.), which is $\sim 0.4$ pc (Barvainis 1987). Hence, we infer that time variability is unlikely to be a main cause of the small $\kappa_{2-10 \mathrm{keV}}$ value. The third possibility, the most plausible one, is that the AGN has a low Eddington ratio $\left(<10^{-3}\right)$, in which case the X-ray to UV luminosity ratio becomes large (Ho 2008). In fact, this could be the case if the SMBH mass in Mrk $463 \mathrm{~W}$ is similar to or larger than that in Mrk 463E, considering that the estimated bolometric luminosity of Mrk $463 \mathrm{~W}$ is $\sim 300$ times smaller than that of Mrk 463E. This can be directly tested by future measurements of the SMBH mass of Mrk 463W. We speculate that this object might be still in an early phase of AGN activity triggered by the merger.

\section{CONCLUSION}

We have analyzed the broadband spectra $(0.4-70 \mathrm{keV})$ of the double-nucleus LIRG Mrk 463 for the first time, observed with NuSTAR, Chandra, and XMM-Newton. An analytical model utilizing the pexmon reflection code and a numerical model utilizing the e-torus model well reproduce the overall spectra. We have determined the absorption column densities of Mrk 463E and Mrk 463W to be $7.5 \times 10^{23} \mathrm{~cm}^{-2}$ and $2.9 \times 10^{23} \mathrm{~cm}^{-2}$, respectively. Significant reflection components are detected in both AGNs, suggesting that their tori are well developed.

The luminosity ratio between X-ray (2-10 keV) and [O IV] $25.89 \mu \mathrm{m}$ of Mrk $463 \mathrm{E}$ is smaller than those of normal Seyferts by a factor of $\sim 5$, indicating that the intrinsic AGN SED is X-ray weak with respect to the UV luminosity. In fact, the bolometric luminosity estimated from the $L^{\prime}$-band $(3.8 \mu \mathrm{m}),[\mathrm{O}$ IV] $25.89 \mu \mathrm{m},[\mathrm{Ne} \mathrm{V}]$ $14.32 \mu \mathrm{m}$ luminosities all yield large bolometric to X-ray luminosity ratios, $\kappa_{2-10 \mathrm{keV}}=110-410$. The Eddington ratio is high, $\lambda_{\mathrm{Edd}}=0.4-0.8$. The large $\kappa_{2-10 \mathrm{keV}}$ value and high Eddington ratio are consistent with the general trend in AGNs found by Vasudevan \& Fabian (2007). These results suggests that Mrk $463 \mathrm{E}$ contains a rapidly growing SMBH, whereas the nucleus is not deeply buried, unlike in typical local ULIRGs.

The bolometric to X-ray luminosity ratio of Mrk 463W, estimated from the $L^{\prime}$-band luminosity, shows an un- 
usually small value, 1-3. We suggest that the Eddington ratio of this $\mathrm{AGN}$ is small $\left(<10^{-3}\right)$, in contrast to Mrk 463E. We speculate that Mrk 463W might be still in an early phase of AGN activity triggered by the merger.

Part of this work was financially supported by the Grant-in-Aid for Scientific Research 17K05384 (Y.U.), $15 \mathrm{~K} 05030$ (M.I.), 15H02070, 16K05296 (Y.T.), and for JSPS Fellows for young researchers (A.T.). C.R. acknowledges financial support from the China-CONICYT fellowship, FONDECYT 1141218, and Basal-CATA PFB-06/2007. Support for this work was provided by the NuSTAR mission, a project led by the California Institute of Technology, managed by the Jet Propulsion Laboratory and funded by the National Aeronautics and Space Administration. This research also made use of data obtained with XMM-Newton, an ESA science mission with instruments and contributions directly funded by ESA Member States and NASA, and with Chandra, supported by the Chandra X-ray Observatory Center, which is operated by the Smithsonian Astrophysical Observatory for and on behalf of NASA.

\section{REFERENCES}

Anders, E., \& Grevesse, N. 1989, Geochim. Cosmochim. Acta, 53, 197

Armus, L., Charmandaris, V., Spoon, H. W. W., et al. 2004, ApJS, 154, 178

Arnaud, K. A. 1996, in Astronomical Society of the Pacific Conference Series, Vol. 101, Astronomical Data Analysis Software and Systems V, ed. G. H. Jacoby \& J. Barnes, 17 Barvainis, R. 1987, ApJ, 320, 537

Bianchi, S., Chiaberge, M., Piconcelli, E., Guainazzi, M., \& Matt, G. 2008, MNRAS, 386, 105

Bianchi, S., Guainazzi, M., \& Chiaberge, M. 2006, A\&A, 448, 499

Dasyra, K. M., Ho, L. C., Netzer, H., et al. 2011, ApJ, 740, 94

Eguchi, S., Ueda, Y., Awaki, H., et al. 2011, ApJ, 729, 31

Fabbiano, G., Wang, J., Elvis, M., \& Risaliti, G. 2011, Nature, 477,431

Gandhi, P., Hönig, S. F., \& Kishimoto, M. 2015, ApJ, 812, 113

Garmire, G. P., Bautz, M. W., Ford, P. G., Nousek, J. A., \& Ricker, Jr., G. R. 2003, in Proc. SPIE, Vol. 4851, X-Ray and Gamma-Ray Telescopes and Instruments for Astronomy., ed. J. E. Truemper \& H. D. Tananbaum, 28-44

Harrison, F. A., Craig, W. W., Christensen, F. E., et al. 2013, ApJ, 770, 103

Heckman, T. M., Ptak, A., Hornschemeier, A., \& Kauffmann, G. 2005, ApJ, 634, 161

Ho, L. C. 2008, ARA\&A, 46, 475

Hopkins, P. F., Hernquist, L., Cox, T. J., \& Kereš, D. 2008, ApJS, 175, 356
Ikeda, S., Awaki, H., \& Terashima, Y. 2009, ApJ, 692, 608

Imanishi, M., \& Saito, Y. 2014, ApJ, 780, 106

Imanishi, M., \& Terashima, Y. 2004, AJ, 127, 758

Imanishi, M., Terashima, Y., Anabuki, N., \& Nakagawa, T. 2003, ApJL, 596, L167

Iwasawa, K., Sanders, D. B., Teng, S. H., et al. 2011a, A\&A, 529, A106

Iwasawa, K., Mazzarella, J. M., Surace, J. A., et al. 2011b, A\&A, 528, A137

Jansen, F., Lumb, D., Altieri, B., et al. 2001, A\&A, 365, L1

Kalberla, P. M. W., Burton, W. B., Hartmann, D., et al. 2005 , A\&A, 440, 775

Kawamuro, T., Ueda, Y., Tazaki, F., Ricci, C., \& Terashima, Y. 2016, ApJS, 225, 14

Komossa, S., Burwitz, V., Hasinger, G., et al. 2003, ApJL, 582, L15

Kormendy, J., \& Ho, L. C. 2013, ARA\&A, 51, 511

Koss, M., Mushotzky, R., Treister, E., et al. 2012, ApJL, 746, L22 -. 2011, ApJL, 735, L42

Liu, T., Wang, J.-X., Yang, H., Zhu, F.-F., \& Zhou, Y.-Y. 2014, ApJ, 783, 106

Mazzarella, J. M., Soifer, B. T., Graham, J. R., et al. 1991, AJ, 102,1241

Mihos, J. C., \& Hernquist, L. 1996, ApJ, 464, 641

Minezaki, T., \& Matsushita, K. 2015, ApJ, 802, 98

Nandra, K., O'Neill, P. M., George, I. M., \& Reeves, J. N. 2007, MNRAS, 382, 194

Nardini, E. 2017, MNRAS, 471, 3483

Oda, S., Tanimoto, A., Ueda, Y., et al. 2017, ApJ, 835, 179

Pereira-Santaella, M., Alonso-Herrero, A., Santos-Lleo, M., et al. 2011, A\&A, 535, A93

Ranalli, P., Comastri, A., \& Setti, G. 2003, A\&A, 399, 39

Ricci, C., Bauer, F. E., Treister, E., et al. 2016, ApJ, 819, 4

-. 2017, MNRAS, 468, 1273

Rigby, J. R., Diamond-Stanic, A. M., \& Aniano, G. 2009, ApJ, 700,1878

Risaliti, G., Imanishi, M., \& Sani, E. 2010, MNRAS, 401, 197

Sanders, D. B., \& Mirabel, I. F. 1996, ARA\&A, 34, 749

Satyapal, S., Vega, D., Heckman, T., O'Halloran, B., \& Dudik, R. 2007, ApJL, 663, L9

Smith, R. K., Brickhouse, N. S., Liedahl, D. A., \& Raymond, J. C. 2001, ApJL, 556, L91

Struck, C. 1999, Phys. Rep., 321, 1

Strüder, L., Briel, U., Dennerl, K., et al. 2001, A\&A, 365, L18

Teng, S. H., Wilson, A. S., Veilleux, S., et al. 2005, ApJ, 633, 664

Teng, S. H., Rigby, J. R., Stern, D., et al. 2015, ApJ, 814, 56

Turner, M. J. L., Abbey, A., Arnaud, M., et al. 2001, A\&A, 365, L27

Vasudevan, R. V., \& Fabian, A. C. 2007, MNRAS, 381, 1235

Veilleux, S., Rupke, D. S. N., Kim, D.-C., et al. 2009, ApJS, 182, 628

Weisskopf, M. C., Brinkman, B., Canizares, C., et al. 2002,

PASP, 114, 1 